\title{
A novel energy management strategy for the ternary lithium batteries based on the dynamic equivalent circuit modeling and differential Kalman filtering under time-varying conditions.
}

WANG, S., STROE, D.-I., FERNANDEZ, C., YU, C., ZOU, C., LI, X. 


\title{
A novel energy management strategy for the ternary lithium batteries based on the dynamic equivalent circuit modeling and differential Kalman filtering under time-varying conditions
}

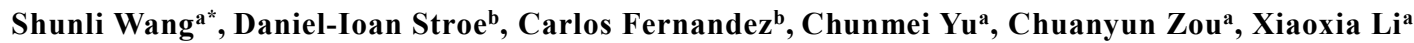 \\ ${ }^{a}$ School of Information Engineering, Southwest University of Science and Technology, Mianyang 621010, \\ China; ${ }^{b}$ Department of Energy Technology, Aalborg University, Pontoppidanstraede 1119220 Aalborg East, \\ Denmark; ${ }^{c}$ School of Pharmacy and Life Sciences, Robert Gordon University, Aberdeen AB10-7GJ, UK.
}

\begin{abstract}
The dynamic model of the lithium battery is a time-varying nonlinear system due to the polarization and diffusion effect inside the battery in the charge-discharge process of the ternary lithium battery. Based on the comprehensive analysis of the energy management methods, the state of charge is estimated for the lithium battery by introducing the differential Kalman filtering method combined with the dynamic equivalent circuit model and nonlinear temperature coefficient. The model simulates the transient response with high precision which is suitable for its high current and complicated charging and discharging conditions. In order to better reflect the dynamic characteristics of the power ternary lithium battery in the step-type charging and discharging conditions, the polarization circuit of the model is differential and the improved model is obtained. As can be known from the experimental verifications, the maximize state of charge estimation error is only 0.022 with the time-varying complex working conditions and the output voltage is monitored simultaneously with the maximum error of $0.08 \mathrm{~V}$ and the average error of $0.04 \mathrm{~V}$. The established model can describe the dynamic battery behavior effectively, which can estimate its state of charge value with considerably high precision, providing an effective energy management strategy for the ternary lithium batteries.
\end{abstract}

Key words: ternary lithium battery; dynamic equivalent circuit modeling; differential Kalman filtering; state of charge estimation; parameter acquisition; nonlinear classification

*Corresponding author: Shun-Li Wang. E-mail address: 497420789@qq.com.

1. Introduction

The internal physical and chemical changes are very complicated for lithium batteries, and it is difficult to apply 
the dynamic battery equation to the actual situation based on the electrochemical theory. In this case, the experimental analysis method is often used, and many different models have been established to predict the behavior of the battery [1]. However, there is no model that can fully simulate the dynamic behavior of the battery accurately under different operating conditions. The state of charge estimation of lithium batteries can make its management system run accurately and efficiently [2-4]. Approximate methods for predicting state of charge include chemical, open circuit voltage, electrochemical impedance, current integration, Kalman filtering and neural network [5], and each of them have their own advantages and disadvantages. As a result, new nonprobabilistic binary linear classification methods are introduced to estimate the state of charge value of lithium batteries from another angle [6-9], which are then used to compensate for the traditional state of charge estimation and increase the state of charge estimating accuracy.

Lithium batteries can be divided into two categories according to the modeling strategy, namely empirical model and electrochemical model [10-15]. The empirical model is a battery dynamic replacement model based on sample data and system identification methods [16], the advantage of which is that its complexity is controllable and modeling cost is low. On the other hand, its disadvantage is that the physicochemical significance of the model parameters is not obvious and its robustness is poor [17]. The electrochemical model is a mathematical expression for obtaining the dynamic characteristics of the battery through the mechanism analysis [18], the advantage of which is that the model has high precision and can reveal the evolution mechanism of the battery characteristics deeply [19]. The disadvantage is that the model has high complexity characteristic, making it not conducive to its engineering application.

According to different establishment mechanisms, the battery model can be divided into simple electrochemical, intelligent mathematical and equivalent circuit model types [20-25]. The electrochemical model is complex and difficult to be applied in the practical products, which is mainly used to assist in the design and manufacture of batteries [26]. The intelligent mathematical model is mainly a neural network model, which can complete battery 
modeling theoretically but requires a lot of actual data for training [27]. Its technical threshold is high and the processing time is long, which limits its practical application [28]. The equivalent circuit model is simple in physical meaning and mathematical expression [29], which is currently widely used. An improved equivalent circuit model is proposed in the battery experimental manual [30], which contains two capacitors and two resistors. The resistor model has one capacitor less than the resistance-capacitance equivalent circuit model [31], the characteristic of which is that the open circuit voltage is considered for the lithium battery. During the charging and discharging process, there will be an error along with the current variation that accumulates over time [32]. The equivalent circuit model internally includes an open circuit voltage source $U_{o c}$, a polarization resistance $R_{p}$, a polarization capacitor $C_{p}$, and an ohmic internal resistance $R_{o}$ and a capacitance $C_{b}$. Since the circuit model covers the characteristics of battery polarization and ohmic internal resistance, it is more accurate for the battery working characteristic expression. Lithium batteries, consisting in high discharge rate, light weight and easy to carry with relatively clean outstanding advantages [33-37], have been applied to various areas of society, such as automobiles, mobile phones, computers and other industries. During the operation of the battery cells, the battery management system will reflect the remaining energy state of the battery [38-44], which is one of its most important factors. Accurate state of charge estimation is of great theoretical value and practical significance for lithium battery development which can make the lithium battery pack safer than usual when used and improve the performance of the battery.

At present, a large amount of researches have been done on the battery state of charge, attracting worldwide concentrations. The commonly used estimation methods include ampere hour integral, open circuit voltage, fuzzy neural network, Kalman filter and so on [45-50]. Starting from the definition, the ampere hour integral method calculates state of charge by the time integral of current, which can only accurately estimate the variation value of the state of charge in a short time, as well as there are problems of not knowing the initial state of charge and the error accumulation effect for a long time [51-55]. Through the one-to-one correspondence between the open- 
circuit voltage and state of charge, the open-circuit voltage of the battery is measured to obtain the state of charge value. As the measured voltage needs to be left standing for a long time [56], the state of charge value cannot be confirmed synchronously. Neural network method is used to estimate the lithium battery state of charge by processing a large number of real-time input and output data of batteries [57-60], which is highly dependent on the training methods and training data when the actual situation has large error influence. The Kalman filter method relies on the establishment of the battery equivalent model and uses the iterative algorithm to obtain the optimal solution in the sense of minimum variance [61-65], which can solve the inaccurate state of charge estimation problem towards the variant initial value and accumulated calculation error. A relatively simple parameter identification method should be used to establish the equivalent model with high precision. Subsequently, in charge and discharge of lithium battery, the dynamic model of the battery is time-varying nonlinear system due to polarization effect and diffusion effect inside the battery. Based on the comprehensive analysis of the above methods, the state of charge of lithium battery is estimated by differential Kalman filter method based on the dynamic equivalent circuit model and temperature coefficient.

2. Mathematical analysis

\subsection{Equivalent modeling}

Electrochemical properties of lithium batteries can be represented by some of the energy storage functions together with electronic components. Meanwhile, general lithium battery equivalent circuit, containing voltage source, resistance and capacitance, can build several classical equivalent circuit model of lithium batteries by these components, such as the internal resistance model, resistance-capacitance model, together with the comprehensive equivalent modeling analysis. The proposed dynamic equivalent model fully considers the response of the circuit to state of charge change and has a high precision with the resistance-capacitance link, which reflects the dynamic effect of the battery and has small calculation amount. The model uses a seriesconnected resistor and resistance-capacitance network to simulate the battery characteristics. As a result, the 
parameters to be identified in this model are ohmic internal resistance, polarization internal resistance, polarization capacitance, which can be realized by the hybrid pulse power characteristic experimental method for its parameter identification. The model equivalent circuit is shown in Fig. 1.

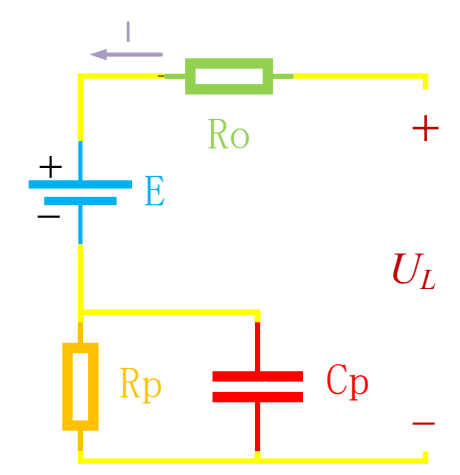

Fig. 1 The dynamic equivalent circuit model

In Fig. 1, the voltage source $E$ is used to represent the open-circuit voltage. $R_{o}$ is used to represent the ohmic internal resistance. $R_{p}$ and $C_{p}$ are used to represent the polarization phenomenon of lithium battery in parallel. $I$ is used to represent the charging and discharging current and $U_{L}$ is used to represent the battery terminal voltage. Afterward, the hybrid pulse power characteristic experimental method is used for parameter identification. A resistance-capacitance circuit is used to simulate the rapid voltage change process of the battery when the current is abrupt together with the slow and stable voltage process that is used to characterize the polarization characteristics of the battery more closely and better simulate its static circuits

\subsection{Differential Kalman filtering}

The differential Kalman filtering method has important advantages in solving small samples, non-linearity and high-dimensional pattern recognition, which can be extended to other machine learning problems such as function fitting. It can analyze data and recognize patterns for classification together with the classical and regressive analysis, which is based on the structural risk minimization principle of statistical learning theory. It can not only classify the linear transforming problems, but also map the input implicitly into high-dimensional feature space for the effective non-linear classification. The expression equations and observation equations of the discrete 
nonlinear system space are shown in equation (1).

$$
\left\{\begin{array}{c}
X_{k+1}=f\left(X_{k}, k\right)+w_{k} \\
Z_{k}=h\left(X_{k}, k\right)+v_{k}
\end{array}\right.
$$

The first part of the equation (1) represents the equation of state, and the second part represents the equation of observation. $k$ is the discrete time point, and $X_{k+1}$ is the $n$-dimensional state vector. $Z_{k}$ is the $m$-dimensional observation vector, and $w_{k}$ and $v_{k}$ are mutually independent Gaussian white noise. In order to apply the Kalman filter, a Taylor expansion is performed on the nonlinear functions $f(*)$ and $h\left(^{*}\right)$ around $X_{k}$. The expansion result is as shown in equation (2).

$$
\left\{\begin{array}{l}
f\left(X_{k}, \quad k\right) \approx f\left(\widehat{X_{k}}, k\right)+\left.\frac{\partial f\left(X_{k}, k\right)}{\partial X_{k}}\right|_{X_{k}=\widehat{X_{k}}}\left(X_{k}-\widehat{X_{k}}\right) \\
h\left(X_{k}, \quad k\right) \approx h\left(\widehat{X_{k}}, \quad k\right)+\left.\frac{\partial h\left(X_{k}, k\right)}{\partial X_{k}}\right|_{X_{k}=\widehat{X_{k}}}\left(X_{k}-\widehat{X_{k}}\right)
\end{array}\right.
$$

As can be known from the calculation process, the values of $A_{k}, B_{k}, C_{k}$, and $D_{k}$ are obtained as shown in the equation (3).

$$
\left\{\begin{array}{l}
A_{k}=\left.\frac{\partial f\left(X_{k}, \quad k\right)}{\partial X_{k}}\right|_{X_{k}=\widehat{X_{k}}} \\
B_{k}=f\left(\widehat{X_{k}}, k\right)-A_{k} \widehat{X_{k}} \\
C_{k}=\left.\frac{\partial h\left(X_{k}, \quad k\right)}{\partial X_{k}}\right|_{X_{k}=\widehat{X_{k}}} \\
D_{k}=h\left(\widehat{X_{k}}, \quad k\right)-C_{k} X_{k}
\end{array}\right.
$$

Then, it can be linearized accordingly as shown in the equation (4).

$$
\left\{\begin{array}{c}
X_{k+1}=A_{k} X_{k}+B_{k}+w_{k} \\
Z_{k}=C_{k} X_{k}+D_{k}+v_{k}
\end{array}\right.
$$

The recursive process of the differential Kalman filtering is obtained by applying the basic equation of Kalman filter to the linearized model as shown in equation (5).

$$
\left\{\begin{array}{c}
\widehat{X_{k+1}^{-}}=f\left(\widehat{X_{k}}\right) \\
\widehat{P_{k+1}^{-}}=A_{k} \widehat{P_{k}} A_{k}^{T}+Q_{k+1} \\
K_{k+1}=\widehat{P_{k+1}^{-}} C_{k+1}^{T}\left(C_{k+1} \widehat{P_{k+1}^{-}} C_{k+1}^{T}+R_{k+1}\right)^{-1} \\
\widehat{X_{k+1}}=X_{k+1}^{-}+K_{k+1}\left[Z_{k+1}-h\left(X_{k+1}^{-}\right)\right] \\
\widehat{P_{k+1}}=\left[I-K_{k+1} C_{k+1}\right] P_{k+1}^{-}
\end{array}\right.
$$


In equation (5), $P$ is the mean square error and $K$ is the Kalman gain. $I$ is an $\mathrm{n} \times \mathrm{m}$ unit array. $Q$ and $R$ are the variances of $w$ and $v$ respectively, which generally do not change along with the time varying system state.

The initial value of the filter and its variance are set as $X(0)=E[X(0)], \quad P(0)=\operatorname{var}[X(0)]$. The filtering step in the $k+1$ cycle is described as follows. First, the state of the current time and the mean square error are predicted from the state $\widehat{X}_{k}$ at $k$ time and the mean square error $\widehat{P_{k}}$ to obtain the prior state $\widehat{X_{k+1}^{-}}$and the prior mean square error $P_{k+1}^{-}$, then the Kalman gain $K_{k+1}$ is calculated for the current time moment. Finally, the state of the current time $K_{k+1}$ is obtained by correcting the prior state, and the mean squared error of the current time is obtained by correcting the a priori mean square error $\widehat{P_{k+1}}$ approximation rather than a solution, which has a higher computational accuracy for the battery state statistics with nonlinear distributions.

\subsection{Iterate state of charge calculation}

The calculation process is described as shown in equation (6).

$$
\left\{\begin{array}{l}
E(t)=U_{L}(t)+R_{p} I(t)+U_{p}(t), I(t)=\frac{U_{p}(t)}{R_{p}}+C \frac{d U_{p}(t)}{d t} \\
S(t)=S\left(t_{0}\right)-\frac{1}{Q_{0}} \int_{t_{0}}^{t} \eta I(t) d t
\end{array}\right.
$$

The three equations are combined and discretized to obtain the state equation as shown in equation (7).

$$
\left\{\begin{array}{l}
x(k \mid k-1)=A_{k} x(k-1)+B_{k} I(k-1)+w_{k} \\
A_{k}=\left[\begin{array}{cc}
1 & 0 \\
0 & e^{-t / \tau}
\end{array}\right], B_{k}=\left[\begin{array}{c}
-t / Q_{0} \\
R_{p}\left(1-e^{-t / \tau}\right)
\end{array}\right]
\end{array}\right.
$$

The observation equation is as shown in equation (8).

$$
\begin{aligned}
y(k) & =h\{x(k-1), i(k-1)\}+v_{k} \\
& =E-R_{o} I(k)-U_{p}(k)+v_{k}
\end{aligned}
$$

After linearizing the obtained Taylor equation, the values of $A_{k}, B_{k}$, and $C_{k}$ are obtained as shown in the equation (9).

$$
\hat{A}_{k}=\left[\begin{array}{cc}
1 & 0 \\
0 & e^{-t / \tau}
\end{array}\right], \hat{B}_{k}=\left[\begin{array}{c}
-t / Q_{0} \\
R_{2}\left(1-e^{-t / \tau}\right)
\end{array}\right], \hat{C}_{k}=\left[\begin{array}{cc}
\frac{\partial U o c}{\partial S O C} & -1
\end{array}\right]
$$

The first step is state prediction, in which the predicted value at time $k$ is calculated as shown in equation (10). 


$$
x(k \mid k-1)=A_{k-1} x(k-1)+B_{k-1} I(k-1)
$$

The second step is the prediction of covariance. By calculating the estimation error of $x(k \mid k-1)$, the covariance matrix of the corresponding $x(k \mid k-1)$ is obtained as shown in equation (11).

$$
P(k \mid k-1)=A_{k-1} \hat{P}_{k-1} A_{k-1}^{T}+Q_{k}
$$

In the third step, the Kalman gain at time $k$ is calculated as shown in equation (12).

$$
K_{k}=P_{k} C_{k}^{T}\left(C_{k} P_{k} C_{k}^{T}+R_{k}\right)^{-1}
$$

The fourth step is the update of the state parameters. The optimal estimated value of the existing state is estimated from the real-time measured factors including the obtained open circuit voltage value $U_{o c}(k)$ to represent $E$ in the equivalent model as shown in the equation (13).

$$
\hat{x}_{k}=x(k \mid k-1)+K_{k}\left[U_{o c}(k)-C_{k} * x(k \mid k-1)\right]
$$

In the fifth step, the noise covariance is updated, which is investigated according to the Kalman gain and the noise covariance of the previous moment as shown in equation (14).

$$
\hat{P}_{k}=\left(1-K_{k} C_{k}\right) P_{k}
$$

The basic iterative calculation process for state of charge estimation is designed. In the calculation, the five steps are continually cycled and the estimated state is continuously updated, so that the estimated value is closer to the true value during the update process. The state of charge estimation formula is shown in the equation (15).

$$
S=\frac{Q_{t}}{Q_{0}} \times 100 \%, S_{I}=\frac{Q_{I t}}{Q_{I 0}} \times 100 \%
$$

In the above expression, the parameter $S_{I}$ is the state of charge value under current $I$ condition, the parameter $Q_{t}$ is the remaining power, and the parameter $Q_{0}$ is the rated capacity. For the continuous time implementation process estimated by the state of charge, it can be represented by the first mathematical expression of the equation. The technical implementation of the battery state of charge estimation for discrete time after its discrete processing can be expressed as shown in the equation (16). 


$$
\left\{\begin{array}{l}
S(t)=S(0)-\int_{0}^{t} \frac{\eta_{i} I(\tau)}{Q_{n}} d \tau \\
S(k+1)=S(k)-\left(\frac{\eta_{i} \Delta t}{Q_{n}}\right) I(k)
\end{array}\right.
$$

The discrete state of charge estimation model shown in the equation (16) facilitates the control of digital systems. In practical applications, discrete state of charge estimation models should be applied. The discrete state space equation of differential Kalman filtering algorithm is given by the process of the ampere hour integration as shown in equation (17).

$$
S(k)=S(k-1)-\frac{\eta}{Q_{n}} I(k-1)+w_{k-1}
$$

The state space description of the equivalent model is located on the observation equation of differential Kalman filtering algorithm, which contains the Kirchhoff laws relation of the equivalent circuit model in time domain as shown in equation (18).

$$
U_{L k}=f\left(S_{k}\right)-I_{k} R_{o}-U_{p k}+v_{k}
$$

Where, $S_{0}$ represents the state of charge value at the initial time, $S_{t}$ represents the state of charge value at time $t, Q_{N}$ represents the rated battery capacity, $I$ represents the current, and the default discharge direction is the positive direction, $\eta$ representing the charging and discharging efficiency. $w$ represents the process noise, which is a gaussian white noise with a mean value of 0 and a variance of $Q$, which is also used to represent the internal error distribution in the system operation. The voltage of the $U_{L}$ representative, $f$ is about the nonlinear function of state of charge, which is introduced to characterize the open circuit voltage. $R_{o}$ and $R_{p}$ the ohmic resistance and the polarization resistance of the model respectively, $\tau$ is used as the resistance-capacitance time constant of the circuit. $v$ is the observation noise with the mean value as 0 and variance value as $R$ that is set as the gaussian white noise, it represents the error distribution appeared in the process of observation. The flow chart of differential Kalman filtering algorithm for state of charge estimation is shown in Fig. 2. 


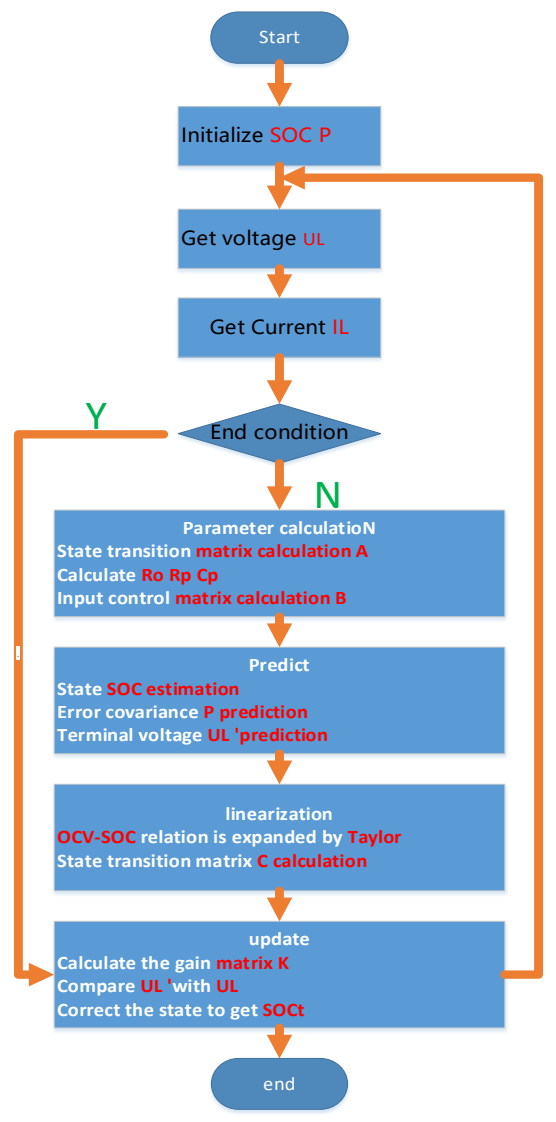

Fig. 2 flow chart of differential Kalman filtering state of charge estimation

At the beginning, the algorithm needs to set as the boundary conditions, that is, the state vector $X$ and error covariance matrix $P$ should be initialized, and the state of charge must be contained in the state vector. Other states are related to the established model, in which $U_{p}$ is considered and characterized. The main part of the algorithm is divided into four stages: parameter calculation, prediction, linearization and update. The content of parameter calculation mainly includes the calculation of circuit element parameters $R_{o}, R_{p}$ and $C_{p}$ in the equivalent model. The significance of state transfer matrix $A$ can be seen from the process recursive equation, which is the driving matrix from the previous state to the next state as shown in equation (19).

$$
X_{k}=A X_{k-1}+B u_{k-1}, X=\left[\begin{array}{l}
S \\
U_{p}
\end{array}\right]
$$

The calculation of state transition matrix $A$ is shown in equation (20).

$$
A=\left[\begin{array}{cc}
1 & 0 \\
0 & e^{-\frac{\Delta t}{\tau}}
\end{array}\right]
$$


The input matrix $B$ is the driving matrix of input influence on state, which is designed as shown in equation (21).

$$
B=\left[\begin{array}{cc}
\frac{\eta \Delta t}{Q_{N}} & 0 \\
0 & R_{p}\left(1-e^{-\frac{\Delta t}{\tau}}\right)
\end{array}\right]
$$

The $\Delta t$ in both equations refers to the sampling time, and the meanings of other parameters have been given in the theoretical analysis process. Therefore, the equation contains the recursive calculation of $S$ and $U_{p}$. In the prediction stage, the reason is given at the prediction point of the error covariance matrix in the state prediction as shown in equation (22).

$$
P_{k / k-1}=A_{k-1} P_{k-1 / k-1} A_{k-1}^{T}+Q_{k-1}
$$

Wherein, $Q$ is the characteristic variance matrix of process noise. The significance of error covariance matrix is that it reflects the probability distribution of the estimated state value in the real value. The smaller the matrix element value is, the more its corresponding state estimation can reflect the real state of the lithium ion batteries. The end voltage prediction is to conducted the end voltage $U_{L}$ of lithium battery at the current moment according to the predicted value of state and Kirchhoff laws circuit equation of model, which is described as shown in equation (23).

$$
U L_{k}=f\left(S_{k}\right)-I_{k} R_{o}-U_{p k}
$$

The super-position observation noise is reduced, because the observation noise changes in real time and is unknown in the actual observation process, according to which its statistical probability distribution rule can be obtained. Linearization is the embodiment of extension in differential Kalman, which extends the range of basic Kalman processing from linear to nonlinear. As for the nonlinear relationship between open circuit voltage and state of charge, the mathematical Taylor expansion is used to obtain the linear expression of the first two terms near the current state of charge estimation value $X_{k}$, and the Jacobian is obtained as the state transition matrix $C$ as shown in equation (24). 


$$
\left\{\begin{array}{l}
U L_{k}=g\left(X_{k}, I_{k}\right)+\frac{\partial g}{\partial X_{k}}\left(x_{k}-X_{k}\right) \\
C_{k}=\frac{\partial g}{\partial X_{k}}=\left[\frac{\partial g}{\partial S_{k}} \quad \frac{\partial g}{\partial U_{p k}}\right]=\left[\frac{\partial\left(U_{O C}-R_{o} i\right)}{\partial S_{k}}-1\right]
\end{array}\right.
$$

Where, the state variable can be obtained nearby and the linear expression is conducted by further transformation as shown in equation (25).

$$
U L_{k}=C_{k} x_{k}+\left(g\left(X_{k}, I_{k}\right)-C_{k} X_{k}\right)
$$

In the equation, when the current state quantity is estimated, the $\left(g\left(X_{k}, I_{k}\right)-C_{k} X_{k}\right)$ term in the equation is a nonrandom action term unrelated to $x_{k}$, thus achieving local linearization. In the process of updating, the gain matrix is calculated first, and then the predicted state quantity is corrected by comparing the observed quantity. As can be known from the above steps, the estimated state value of $\hat{X}_{k}$ at time point $k$ is obtained.

3. Experimental analysis

\subsection{Program and platform design}

The parameter identification of equivalent model of ternary lithium battery needs to be completed by the experiments. The experimental platform is formed by the ternary lithium batteries, battery test equipment (BTS 750-200-100-4), high and low temperature experiment box (DGBELL) and a computer, according to which the experimental platform is set up and the hybrid pulse power characteristic experiments are carried out under different working conditions together with the main discharge verification tests. Since lithium batteries are generally charged at constant current and then at constant voltage, the state of charge at the time of charging is relatively easy to be estimated. Therefore, only the experiment of parameter identification of discharge direction is conducted, namely compound pulse power experiment. The equipment of BTT-331C made by Dongguan Bell Experimental Equipment corporation is used in the experimental test. The experimental equipment also include the charge-discharge facilities made by Shenzhen Xinwei New Energy Technology corporation. The experimental platform is shown in Fig. 3. 


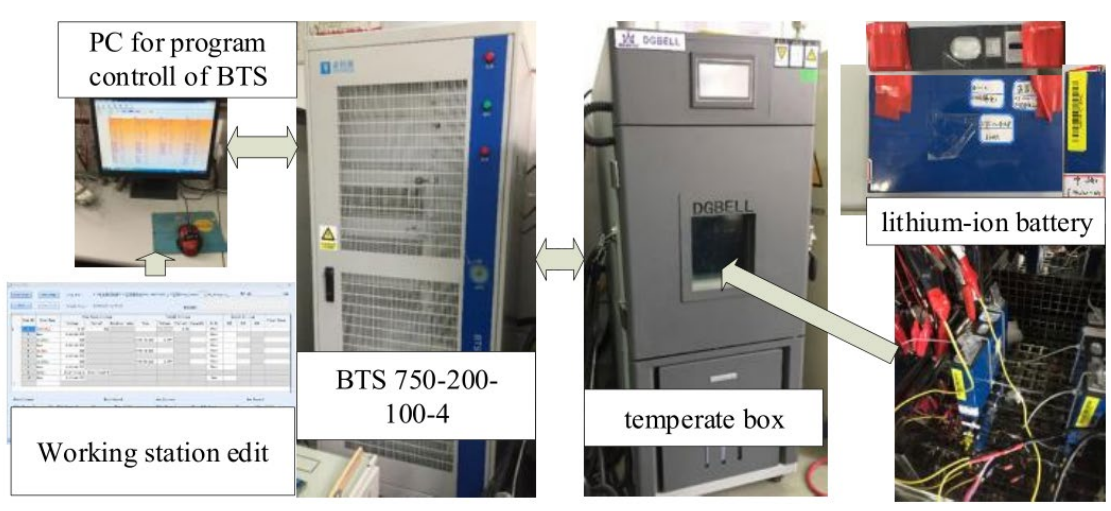

Fig. 3 The experimental platform

In the experiment, the battery capacity tester is used to charge and discharge the lithium batteries. As in the experimental procedure, the support vector machine is used to train the data, obtaining a general mathematical equation model through mathematical modeling, which is then substitute into the calculation process. The corresponding state of charge prediction value is obtained, which is finally compared and matched with the experimental value and the predicted value. At the same time, the initial state of charge value is set to be 0 and $C=1$. In this experiment, the ternary lithium batteries (CFP50AH) are selected as the experimental object, the basic technical parameters of which are shown in Tab.1.

Tab.1 Basic technical parameters of the test battery samples

\begin{tabular}{|c|c|}
\hline Factor & parameter \\
\hline Size: length * width * height $/ \mathrm{mm}$ & $200 * 80 * 180$ \\
\hline Rated voltage/V & 3.7 \\
\hline Maximum load current $/ \mathrm{A}$ & $1.5 \mathrm{C}$ \\
\hline Charge cut-off voltage $/ \mathrm{V}$ & 4.15 \\
\hline Discharge cutoff voltage $/ \mathrm{V}$ & 3.0 \\
\hline Working temperature $/{ }^{\circ} \mathrm{C}$ & $-15 \sim 70$ \\
\hline Rated capacity $/ \mathrm{Ah}$ & 4.0 \\
\hline
\end{tabular}

The incubator is a three-layer independent temperature control high and low temperature test chamber (DGBELL BTT-331C) of Dongguan Bell Experimental Equipment Co., Ltd., and the experiments are carried out at a constant temperature of $25^{\circ} \mathrm{C}$.

The purpose of compound pulse power experiment is to get the parameters of equivalent circuit model corresponding to different state of charge levels. It is investigated at room temperature ( 25 degrees) on a full 
charge and hold the battery after the first pulse discharge for 10 seconds, and then the batteries are set stand for 40 seconds. It should be treated again to the battery pulse charge for 10 seconds, and the pulse discharge current value in the experiment with the charging treatment is $1 \mathrm{C}$ current rate before it is discharged to the point where the state of charge decreased $10 \%$. The 40 minutes interval should be conducted after each experiment step to let the battery electrochemistry reaction inside be equilibrium, so the process in these different state-of-charge points can be investigated to do the same steps for experiments in order to get the response data of the voltage and current. The discharge current is assumed to be negative, the experimental result of which is shown in Fig. 4.

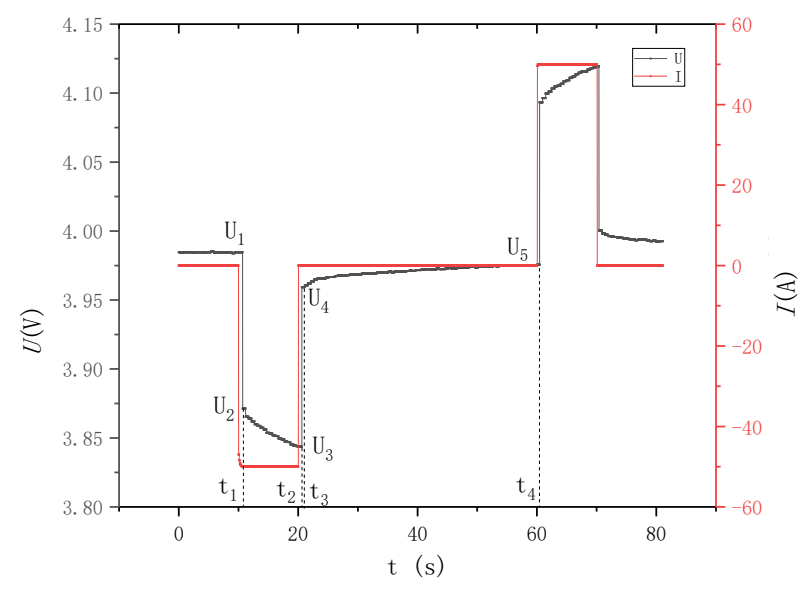

Fig. 4 hybrid pulse power characteristic experimental voltage and current curve

It can be seen from the curves in the above two figures that the current value and voltage value at $t_{1}$ both have abrupt changes and increase sharply, which reflects the internal resistance characteristic of model. As can be known from $t_{2}$ to $t_{3}$, the terminal voltage $U_{L}$ value shows a gradually decreasing change. According to Kirchhoff laws of circuit, the polarization capacitor $C_{P}$ partial voltage makes the terminal voltage gradually decrease, and the polarization voltage $U_{p}$ increases gradually from $0 \mathrm{~V}$. Similarly, in the $t_{4}$ to $t_{5}$ process, it is regarded as the zero-input response process with gradually decreasing $C_{p}$ capacity along with the $U_{p}$ gradually decreases, and the $U_{L}$ gradually increases and finally equals to the open-circuit voltage value. Finally, the whole process of the hybrid pulse power characteristic test along with pulse charging and discharging treatment can be obtained when the battery voltage current curve and corresponding resistance-capacitance link can be applied into the corresponding 
model, showing the polarization effect of the battery. Since battery features of the model and the actual is very association, a simple first order resistance-capacitance circuit is zero and the zero input state equation can complete the parameter identification that is established in advance.

Through the above experiments and modeling work, the battery is transformed into an equivalent complex electrochemical reaction system. In short, the equivalent model refers to the battery system to load the same current, according to which the established equivalent model can output the actual battery voltage by the same mathematical treatment or similarly. The voltage of the model is accurate and the actual error is small, demonstrating the high precision of the model. The model parameter identification is investigated in the state of charge estimation under the working condition influence of the model parameter values given or known including battery capacity, charging and discharging rate, cell aging degree and the environmental temperature. The electrochemical and physical knowledge are used together with the data analysis software as accurately as possible to calculate or fit out all the parameters of the selected model under the complex working conditions by introducing the values of dependent and independent variables into the calculation, according to which the given system is the battery equivalent model process combined with its parameter identification. Similarly, when the conditions are changed gradually in the experimental environment, corresponding model parameters can be calculated one by one. When many condition-results are obtained, the variation rules among variables can be acquired to know the change rule of the open circuit voltage of the model. In addition, it is easy to know that the open circuit voltage is mainly affected by the state of charge and temperature of the battery through the external characteristic experiments. When the temperature is constant, the function of the open circuit voltage changing along with state of charge can be obtained by testing the open circuit voltage of the battery at different state of charge level points by Matlab data fitting.

In the equivalent model, there are four model parameters to be identified: open circuit voltage $U_{o c}$, internal resistance $R_{o}$, polarization resistance $R_{p}$ and polarization capacitor $R_{p}$. Under certain aging degree and temperature, 
these four parameters are mainly affected by the state of charge level. Through the hybrid pulse power characteristic experiment described above, it is easy to get the curve relationship between open circuit voltage and state of charge, including the parameter identification of internal resistance $R_{o}$. When conducting a hybrid pulse power characteristic experiment, the discharge maintenance starts at time point $t_{1}$. At this time moment, the internal resistance $R_{o}$ is obtained by dividing the voltage difference before and after the mutation by the discharge current and the polarization resistance $R_{p}$ can be identified. According to the experimental analysis, the battery stands for 40 seconds from $t_{4}$ to $t_{5}$, during which the current is zero. By analyzing the resistance-capacitance circuit, it can be concluded that the polarization voltage $U_{p}$ is the zero input response and the response expression of the circuit is shown in equation (26).

$$
\left\{\begin{array}{l}
U_{L}=U_{O C}+U_{p} \\
U_{p}=U_{p}(0) e^{-1 / t}
\end{array}\right.
$$

Among them, $U_{L}$ is the terminal voltage of the battery that can be obtained from the voltage response curve, in which $U_{O C}$ is the open circuit voltage of the battery. The response curve of polarization voltage can be obtained according to equation (26). The initial value of polarized voltage can be calculated at the end of discharge voltage, according to which the polarization voltage response curves can be obtained by investigating the least square fitting treatment together with the time parameters in the model.

The battery cell has been standing for a long time before each hybrid pulse power characteristic experiment, so it can be considered that the polarization effect of the resistance-capacitance circuit has disappeared. By observing the voltage response curve, the response of the circuit from $t_{2}$ to $t_{3}$ can be regarded as the zerostate response. In this case, the electric response is obtained as shown in equation (27).

$$
\left\{\begin{array}{l}
U_{L}=U_{O C}+I R_{o}+U_{p} \\
U_{p}=U_{p}(0) e^{-1 / t}
\end{array}\right.
$$

The same way can be conducted along with the above time parameters in the model, according to the equation (27) and the polarization resistance resulting from the curve fitting. The circuit time parameter can 
be divided by the polarization resistance, according to which the polarization capacitance can be obtained. According to the above parameter identification method, the parameter identification results are obtained shown in Tab.2.

Tab.2 model parameter identification results

\begin{tabular}{|c|c|c|c|c|}
\hline $\mathrm{S}$ & $\mathrm{Ro}(\Omega)$ & $\mathrm{Cp}(\mathrm{F})$ & $\mathrm{Rp}(\Omega)$ & $\mathrm{E}(\mathrm{V})$ \\
\hline 1.000000 & 0.0012 & 73021.9735 & 0.0062532 & 4.18 \\
\hline 0.897584 & 0.0012 & 38779.0975 & 0.0016404 & 4.05 \\
\hline 0.795148 & 0.0012 & 31237.4083 & 0.0019628 & 3.94 \\
\hline 0.692691 & 0.0016 & 38306.3389 & 0.0016788 & 3.84 \\
\hline 0.590254 & 0.0016 & 28407.8836 & 0.0011348 & 3.74 \\
\hline 0.487797 & 0.0012 & 77466.2222 & 0.0017468 & 3.65 \\
\hline 0.385340 & 0.0016 & 66796.6261 & 0.0015792 & 3.62 \\
\hline 0.282884 & 0.0012 & 48324.2877 & 0.0017932 & 3.58 \\
\hline 0.180447 & 0.0016 & 69167.8689 & 0.0015940 & 3.52 \\
\hline 0.077990 & 0.0016 & 22738.2283 & 0.0002188 & 3.43 \\
\hline 0.000000 & 0.0024 & 22738.2283 & 0.0002188 & 3.26 \\
\hline
\end{tabular}

\subsection{Model accuracy verification}

In order to verify the effect of parameter identification and the accuracy of the equivalent model, the equivalent circuit model is built in Simulink, and the accuracy of the model is verified under the hybrid pulse power characteristic experimental current condition, constant current condition and Beijing bus dynamic stress test condition respectively. Experimental IL module is used as the input load current condition, aiming to calculate state of charge value at each time moment by ampere-hour integration method, according to which the input state 
of charge is inputted into the LOOKUP TABLE module. The online identification of four parameters is completed by using the piecewise linear interpolation method. At last, the designed s-function is used to calculate and output the closed voltage $U_{L}$ at each time moment, and then it is compared with the experimental voltage corresponding to the experimental load current $I_{L}$ to analyze the accuracy of the model and complete the verification of the model. According to the Beijing bus dynamic stress test operating current data and calculation model, the comparison between the simulation and the actual terminal voltage curve are shown in Fig. 5.

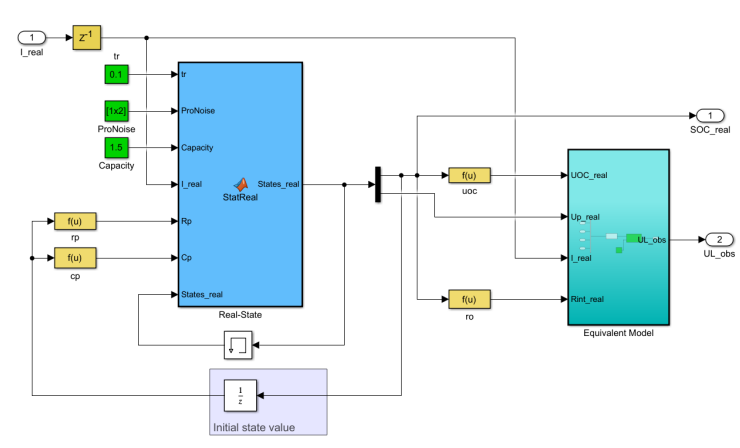

(a) End voltage verification schematic diagram

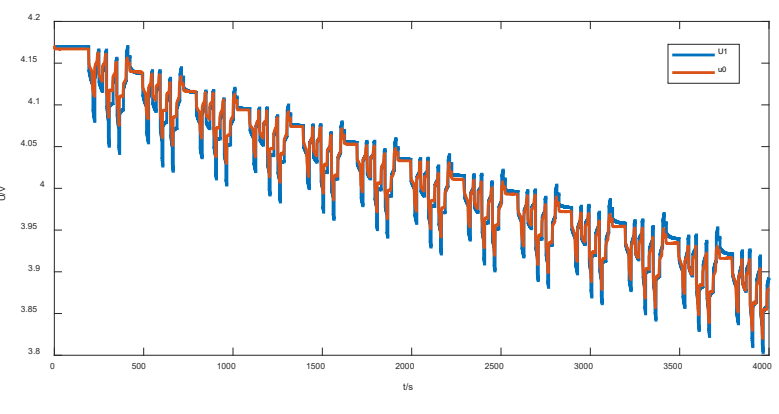

(b) Camparative terminal voltage curve

Fig. 5 Experimental operating current data

As can be known from the experimental results, the simulation value of the battery terminal voltage of the equivalent model under BeijingBeijing bus dynamic stress test current condition and the same voltage of the current conditions of the actual value of contrast model, the voltage of the overall model of the simulation curve can accurately follow the change of the actual voltage. In each current mutation point, the estimated voltage and the actual voltage present the same change trend, but in some point voltage of the mutations, the actual voltage variation exist large deviation due to the resistance identification model only considers the effect of the state of charge and temperature, which ignores the factors such as charge- discharge rate, internal resistance identification inaccuracy result. In addition, part of the simulation model of zero input and zero state response cannot accurately reflect the change of the actual voltage of the battery, because of ignoring these factors. The equivalent model considers the selected polarization capacitance and resistance in the stage of building model with piecewise linear 
interpolation method instead of the actual $C_{P}$ and $R_{P}$ changing with the state of charge. A relatively simple parameter identification method is used to establish the equivalent model with high precision with the maximum error of $0.08 \mathrm{~V}$ and the average error of $0.04 \mathrm{~V}$.

\subsection{Iterate calculation construction}

According to the experimental and theoretical analysis, the state of charge estimation algorithm is constructed by using experimental data based on the accurate modeling of the battery. The main raw data can be used under different working conditions of the experimental data in order to test the accuracy of the proposed algorithm, and the stability and applicability are analyzed by using the constant current conditions respectively. And then, the Beijing bus dynamic stress test condition is compared to the simulation and experimental test, in which the comparison between ampere-hour integral, differential Kalman filtering and traditional Kalman filtering traditional Kalman filtering algorithm estimation effect is investigated to test the feasibility and validity of the algorithm. The realtime state verification is conducted by the ampere-hour integral approach with minimum time interval as the standard, which is used to compare and verify the state output effect under each condition.

The experimental data is adopted to simulate the actual operation to define the data in the MATLAB environment effective digit processing, aiming to obey the normal distribution of random noise together with the gaussian white noise, the mean zero and variance of $R$. The superimposed on the input data is used to simulate the actual observation of terminal voltage value, in which the data is taken as a voltage contrast state of charge estimation update and input analog observation noise voltage using the differential Kalman filtering algorithm based on equivalent circuit model in both of the forecast and update processes to get the state of charge estimation output. Based on the original current data and the ampere hour integral method, a process noise matrix with variance matrix $Q$ is superposed to obtain the state vector, which is used as the real vector. The accuracy and stability of the model and algorithm are verified by comparing the simulated output state of the algorithm with the simulated real state. 
For the iterative calculation process of the differential Kalman filtering algorithm, the boundary conditions are set and given initial quantity. Including the state of the initial amount $X_{0}$, the charge state initial value $S_{0}$ and $U_{p 0}$ polarization voltage are set with initial values in the model. In practice, the initial value of the state of charge can be recorded by the management system before the state quantity in the case of battery put on hold for a long time, which can be then used to improve the static charge state estimation accuracy to get the initial value. Just using the battery before starting the polarization effect is not obvious, so the polarization voltage can be thought as the initial value of zero. The initial value $P_{0}$ of the error covariance matrix is determined from the $X_{0}$ error of the initial state. In application, the initial value should be kept as small as possible to speed up the tracking speed of the algorithm. Two important parameters are process noise variance matrix $Q$ and observation noise variance matrix $R$. It can be seen from the theoretical equation derivation of differential Kalman filtering that it plays a key role in the estimation effect of the algorithm, because it directly affects the size of Kalman gain matrix $K$ and the value of error covariance matrix $P$. As mainly comes from the measurement error distribution of experimental instruments and sensors, the expression of the observation noise variance can be obtained as shown in equation (28).

$$
R_{k}=E\left(v_{k}^{2}\right)=\sigma_{v}^{2}
$$

Generally, the two states in the system are not correlated and the covariance is zero, so only the variance on the diagonal has a value. The relationship between the process variance of the system and the covariance of process noise can be obtained as shown in equation (29).

$$
Q_{k}=\left[\begin{array}{cc}
E\left(\omega_{1 k}^{2}\right) & E\left(\omega_{1 k} \omega_{2 k}\right) \\
E\left(\omega_{2 k} \omega_{1 k}\right) & E\left(\omega_{2 k}^{2}\right)
\end{array}\right]=\left[\begin{array}{cc}
\sigma_{\omega 1,1}^{2} & \sigma_{\omega 1,2}^{2} \\
\sigma_{\omega 2,1}^{2} & \sigma_{\omega 2,2}^{2}
\end{array}\right]
$$

The process noise variance $Q$ is mainly derived from the system noise and the error of the established equivalent model. It is difficult to obtain it by the theoretical methods or means, but a reasonable value range can be obtained through continuous debugging in simulation and it is of small quantity. 
(1) comparison of operating conditions

In order to verify the applicability and stability of the algorithm, the input parameters in different working conditions are used to observe the estimated accuracy of the algorithm. In this simulation, two operating conditions are used, in which one is the constant current condition with shelving stage and the other is in the Beijing bus dynamic stress test condition. For constant-current condition, only a constant value needs to be set in the program. In order to increase the complexity of the condition, two shelving stages are added into the constant-current input list to test the tracking effects of Ampere hour and differential Kalman filtering. The following code is generated for the current.

I_real $=1.5 *^{*}$ ones $(1, \mathrm{~N})$;

I_real $(\operatorname{ceil}(\mathrm{N} / 4)$ : $\operatorname{ceil}(\mathrm{N} / 3))=0$;

I_real $\left(\operatorname{ceil}(\mathrm{N} * 2 / 3)\right.$ : ceil $\left.\left(\mathrm{N}^{*} 3 / 4\right)\right)=0$;

Experimental data is required for Beijing bus dynamic stress test condition pair algorithm simulation, which shows the current input module in Simulink environment. The upper part is the ampere integral module, whose output is the actual capacity change of Beijing bus dynamic stress test condition, while the lower part is the condition data output module. It outputs the working current to the workspace at the minimum time interval of actual measurement sampling. The data in the workspace is timeseries type and the current data in it needs to be extracted, in which the data extraction and transformation is performed in the main program as shown in the following code.

Function main (S_est_init)

Sim Beijing bus dynamic stress test_working condition;

$\mathrm{I}=$ - (current.data) ';

differential Kalman filtering S estimation (S_est_init, I);

The End 
The Beijing bus dynamic stress test working condition is the module name of Simulink, in which Current is the time series data including current output, differential Kalman filtering S Estimation is the algorithm program module, and its input is the $\mathrm{S}_{-}$est_init initial value of state of charge estimation and current data. The current data input to the differential Kalman filtering algorithm is based on model program module, other parameters of the initial value within the program have been identified, the program final output with minimum time interval for 10 times the length of the sampling time ampere-hour integral and differential Kalman filtering state of charge estimation curve.

(2) algorithm comparison

Firstly, the algorithm simulation was realized by writing scripts in MATLAB, and traditional Kalman filtering algorithm was added to compare the output effect with each other. The specific implementation process met the requirements of the process, and the main simulation program is shown as follows.

Function main (S_est_init, Work_modes)

If Work_modes $==1$

Sim Beijing bus dynamic stress test_workingcondition;

$\mathrm{I}=-(\text { current.data) })^{\prime *} 1.5 / 50$;

Elseif Work_modes $==2$

$\mathrm{N}=60001$;

$\mathrm{I}=1.5 *$ ones $(1, \mathrm{~N})$;

I $(\operatorname{ceil}(\mathrm{N} / 4): \operatorname{ceil}(\mathrm{N} / 3))=0$;

I $\left(\operatorname{ceil}\left(\mathrm{N}^{*} 2 / 3\right)\right.$ : $\left.\operatorname{ceil}\left(\mathrm{N}^{* 3 / 4}\right)\right)=0$;

The else

Disp (" input parameter error!" );

The end 
The end

The program takes the minimum time interval of 10 times as the sampling time, and simulates three methods of ampere-hour integral, differential Kalman filtering and traditional Kalman filtering to estimate state of charge, aiming to compare the tracking of the three methods to the actual state of charge over time. The program outputs both the state of charge change curve and the estimation error curve, so that the tracking effect and error change of the three methods can be intuitively compared with each other. Based on script implementation, the Simulink modular simulation is built. Its implementation process and script program is basically the same, which uses different modules to replace the script code block by using the integration advantages of Simulink environment itself in the graphical user interface to achieve the same simulation effect. The aim of this treatment is to ensure that the running logic is unchanged under the more intuitive presentation of the algorithm running process and the established model is shown in Fig. 6.

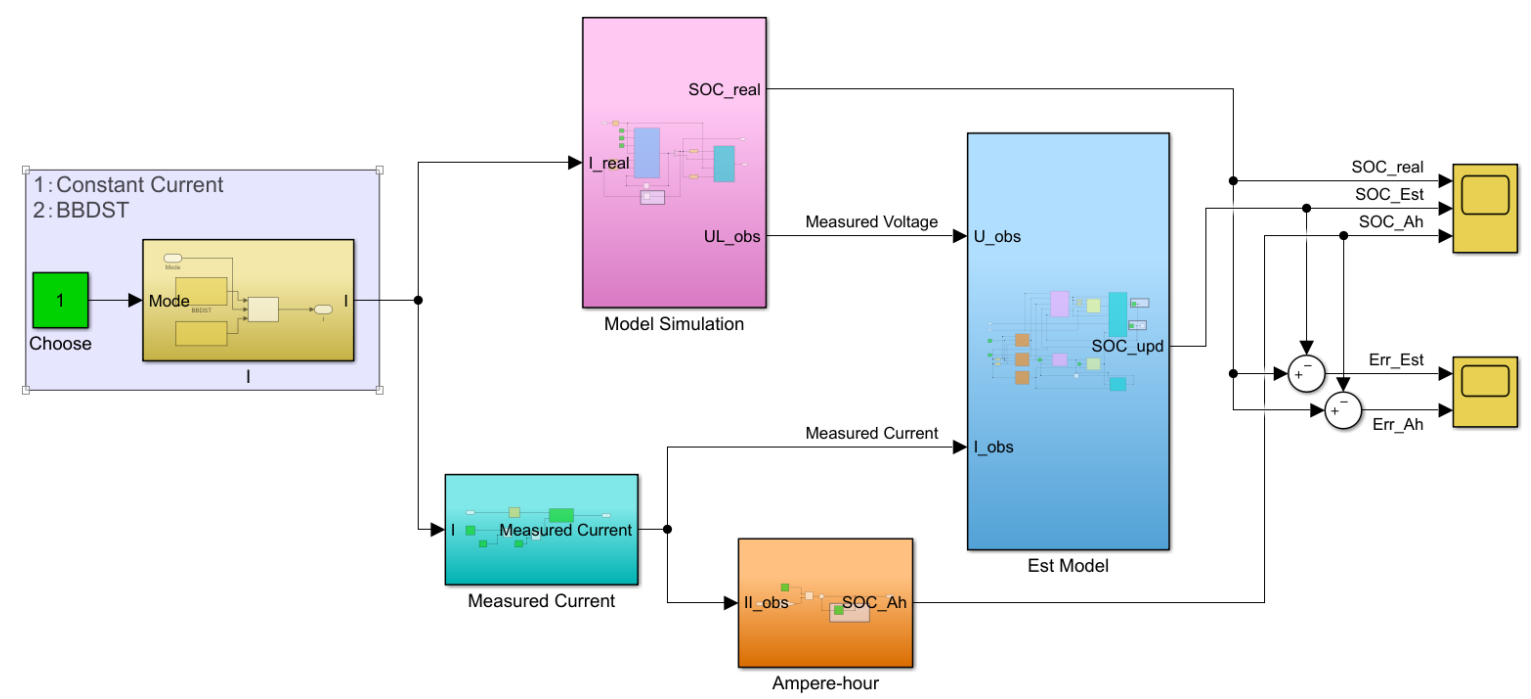

Fig. 6 Simulink algorithm simulation

The diagram is constructed with the experiment data as the input current, the state of charge prediction is obtained by its prediction module, which are calculated by the forecast treatment by using the model parameters. Meanwhile, the equivalent model is constructed to predict the quantity of $U_{p}$ completion status. Subsequently, the obtained output voltage of predicted values can be used as the input of the differential Kalman filtering algorithm 
update module at the same time, including the update module output state correction S_upd, Up_upd and error covariance matrix P_upd update that are taken as the basis of the forecast. In the simulation, the ampere-hour integral result of the minimum time interval as well as the actual current data is taken as the reference value of the real-time state of charge estimation, and its stability is evaluated under the operating condition by comparing the estimation effect of Ampere hour and differential Kalman filtering when the sampling period is 10 times the hourly shift.

The internal structure of model is realized in the Simulink simulation, where the state quantity $U_{p}$ needs to accept the last estimated value Up_upd as the basis for the next state prediction and recursion, and at the same time, it is quite convenient to directly input the predicted value Up_pre to the corresponding position of resistancecapacitance circuit in the circuit model that is taken as the current polarization voltage for terminal voltage prediction. As can be seen from the experimental results, more modular implementation of each part of the function, more clear and complete structure can be obtained for the distinct hierarchy.

\subsection{Main charging condition effect}

The lithium battery main charging experiment design process is shown in Tab.3.

Tab.3 Lithium battery charging experiment step design

\begin{tabular}{|l|l|l|l|}
\hline Step number & Work type & Work step parameters & Closing condition \\
\hline 1 & Constant current discharge current & current: $10 \mathrm{~A}$ & voltage: $2.5 \mathrm{~V}$ \\
\hline 2 & Shelving time & time: $0: 20: 00.000$ & time: $1: 30: 00.000$ \\
\hline 3 & Constant current charging current & current: $2.5 \mathrm{~A}$ & \\
\hline 4 & Shelving time & time: $0: 20: 00.000$ & time: $0: 45: 00.000$ \\
\hline 5 & Constant current discharge current & current: $1.5 \mathrm{~A}$ & time: $0: 30: 00.000$ \\
\hline 7 & Shelving time & time: $0: 10: 00.000$ & \\
\hline
\end{tabular}




\begin{tabular}{|l|l|l|l|}
\hline 8 & Constant current charging current & current: $3.5 \mathrm{~A}$ & time: $1: 00: 00.000$ \\
\hline 9 & Constant current charging current & current: $2.5 \mathrm{~A}$ & time: $1: 00: 00.000$ \\
\hline 10 & Shelving time & time: $0: 20: 00.000$ & time: $0: 45: 00.000$ \\
\hline 11 & Constant current discharge current & current: $1.5 \mathrm{~A}$ & \\
\hline 13 & $\begin{array}{l}\text { Shelving time } \\
\text { charging }\end{array}$ & time: $0: 10: 00.000$ & current: $0.5 \mathrm{~A}$ \\
\hline
\end{tabular}

In the charging process of the lithium battery, it is very necessary to completely discharge the residual lithium battery in advance, which satisfies the initially set $S=0$. It is generally considered that the lithium battery is completely discharged when the voltage of the lithium battery drops to $2.5 \mathrm{~V}$. After a constant current charging process with different currents for a certain time period, the lithium battery is gradually charged until the state of charge is about 1 to stop charging. Since the system cannot recognize the state of charge value of the lithium battery at each moment, the set stop condition is changed so that the power supply is stopped when the battery voltage reaches $3.65 \mathrm{~V}$ as shown in Fig. 7.

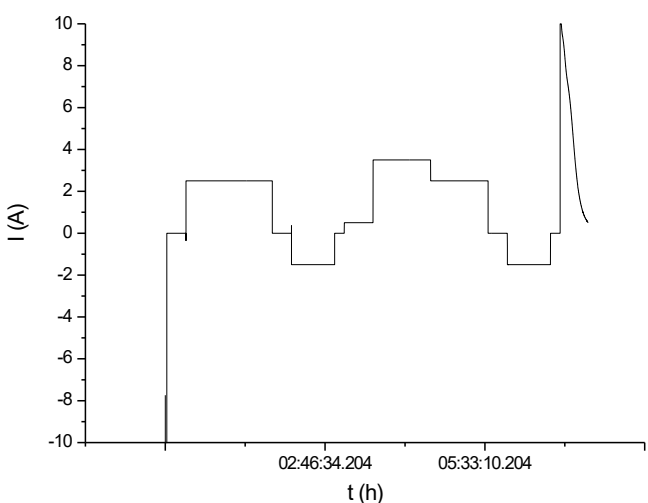

a) Current

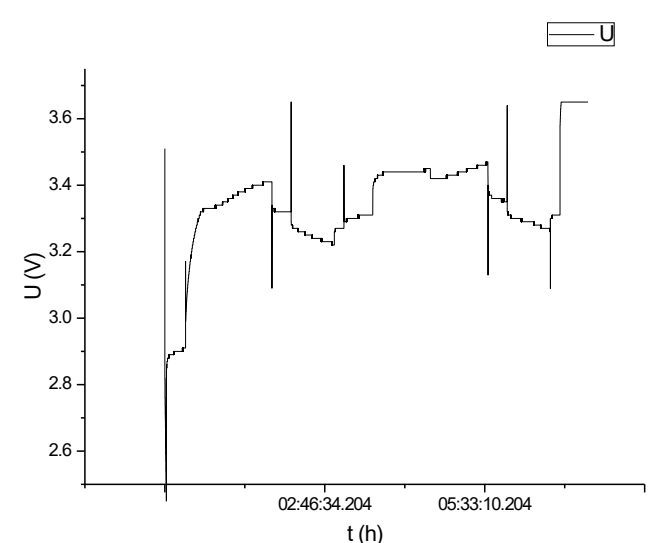

b) voltage 


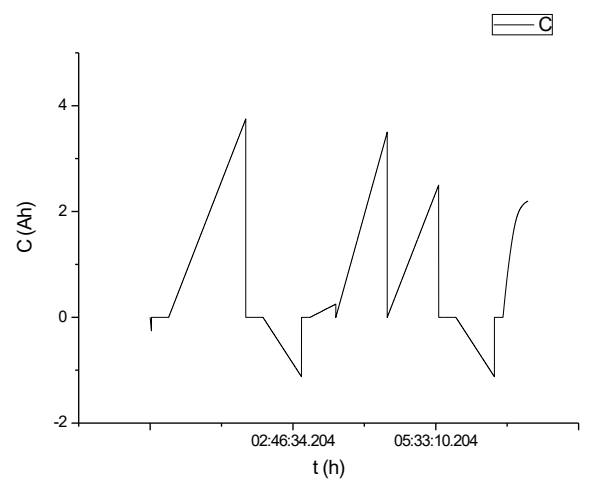

a) capacity

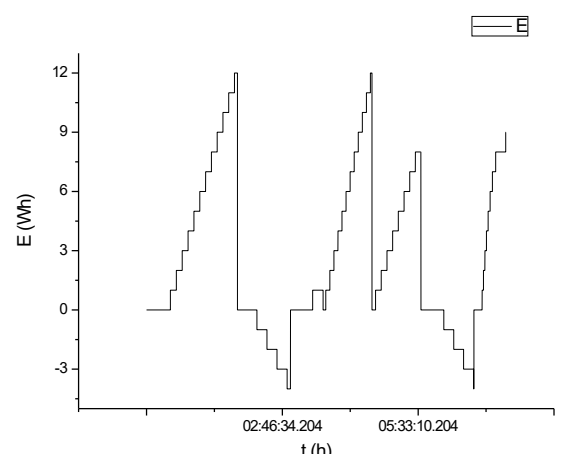

b) energy

Fig. 7 Charging process parameter variation waveform

Throughout the process, since the main purpose is to charge the lithium battery, both the capacity and the energy waveform fluctuate when the constant current charge and discharge interval is performed. The capacity generally only considers the number of charges in the material reaction, and the difference in energy and capacity is that it also contains the voltage factor. The higher the voltage platform, the higher the energy. The trend of the two factors following the time is roughly the same over time.

\subsection{Main discharging condition effect}

The design process of lithium battery discharge experiment is shown in Tab.4.

Tab.4 Lithium battery discharge experimental steps design

\begin{tabular}{|l|l|l|l|}
\hline Step number & Work type & Work step parameters & Closing condition \\
\hline 1 & Constant current constant voltage charging & $\begin{array}{l}\text { voltage: } 3.65 \mathrm{~V} \\
\text { current: } 10 \mathrm{~A}\end{array}$ & current: 0.5 A \\
\hline 2 & Shelving time & time: 0:20:00.000 & \\
\hline 3 & Constant current discharge & current: $1 \mathrm{~A}$ & voltage: $2.5 \mathrm{~V}$ \\
& & time: 0:20:00.000 \\
\hline 4 & Constant current discharge & current: 2 A & $\begin{array}{l}\text { voltage: } 2.5 \mathrm{~V} \\
\text { time: } 0: 20: 00.000\end{array}$ \\
\hline 5 & Constant current discharge & current: $4.5 \mathrm{~A}$ & $\begin{array}{l}\text { voltage: } 2.5 \mathrm{~V} \\
\text { time: } 0: 20: 00.000\end{array}$ \\
\hline 6 & & $\begin{array}{l}\text { voltage: } 2.5 \mathrm{~V} \\
\text { time: } 0: 20: 00.000\end{array}$ \\
\hline 7 & Constant current discharge & current: $0.5 \mathrm{~A}$ & voltage: $2.5 \mathrm{~V}$ \\
\hline
\end{tabular}




\begin{tabular}{|l|l|l|l|}
\hline & & & time: 0:20:00.000 \\
\hline 8 & Cycle & $\begin{array}{l}\text { start work: 3 } \\
\text { cycle times: } 3\end{array}$ & \\
& & & \\
\hline
\end{tabular}

The discharge of the lithium battery is to discharge the fully charged battery to zero. First, the lithium battery is fully charged by a constant current and constant voltage charging method. Several different constant currents are set for cyclic discharge. Through calculation and analysis, it can be known that under the set known conditions, the lithium battery can be completely discharged by three cycles. That is, the cutoff condition is that the lithium battery voltage is $2.5 \mathrm{~V}$. The waveform of the current and voltage change is shown in Fig. 8 .

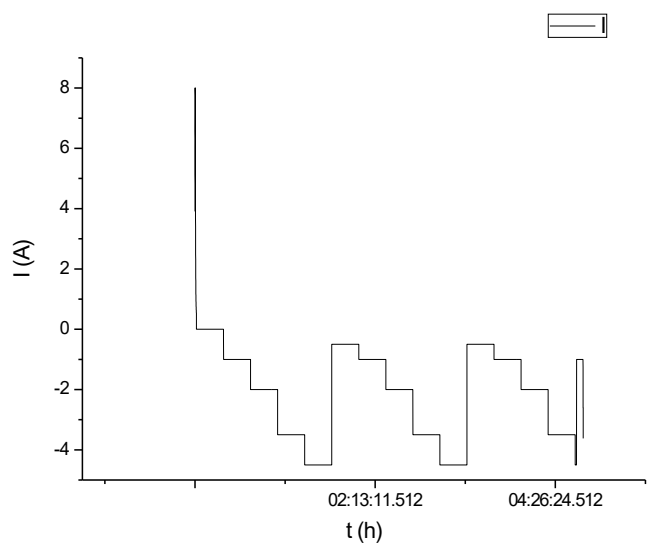

a) Current

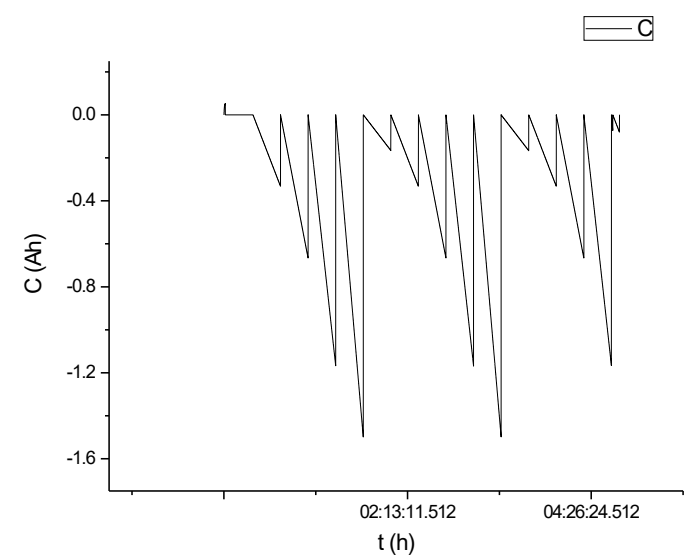

c) capacity

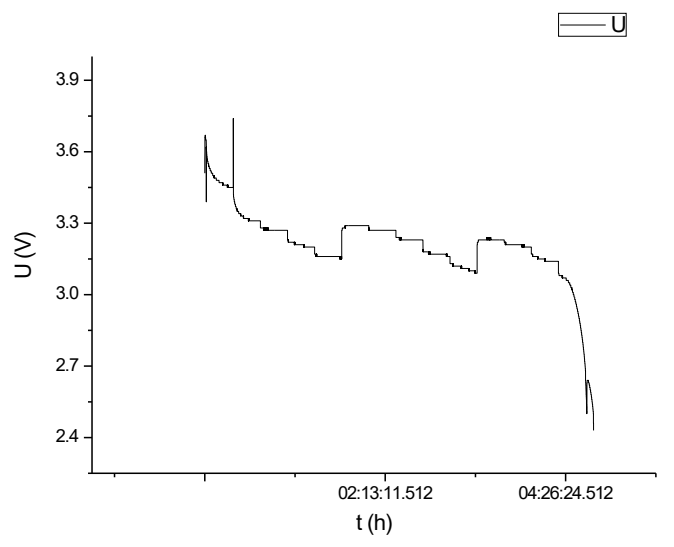

b) voltage

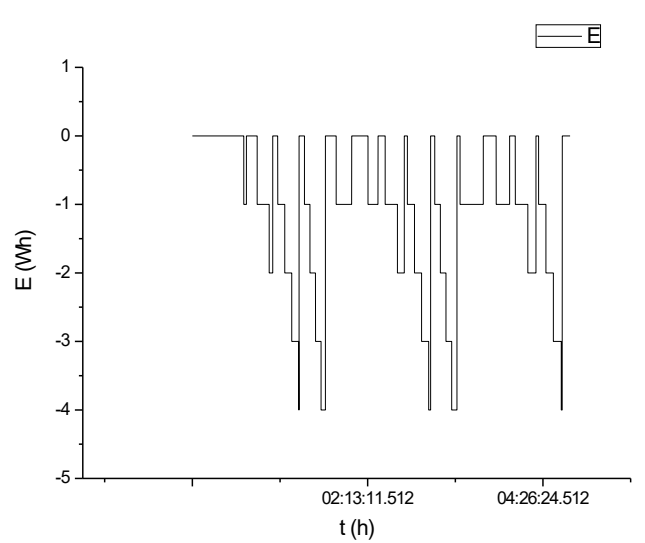

d) energy

Fig. 8 Discharge process parameter variation waveform 
When the lithium battery is discharged as a whole, the voltage change waveform shows a downward trend.

The trend of capacity and energy is roughly the same.

\subsection{Complex validation and analysis}

The constant current conditions of algorithm simulation results can be obtained, in which the blue curve is a true value, state of charge red curve for state of charge estimation results using the Ampere hour integral method, the green curve for state of charge estimation of differential Kalman filtering algorithm, the orange curve for state of charge estimation results of traditional Kalman filtering algorithm, graph curves of upper column for state of charge value, the estimation algorithm of the bar under the conditions of the error of curve Fig. 9.
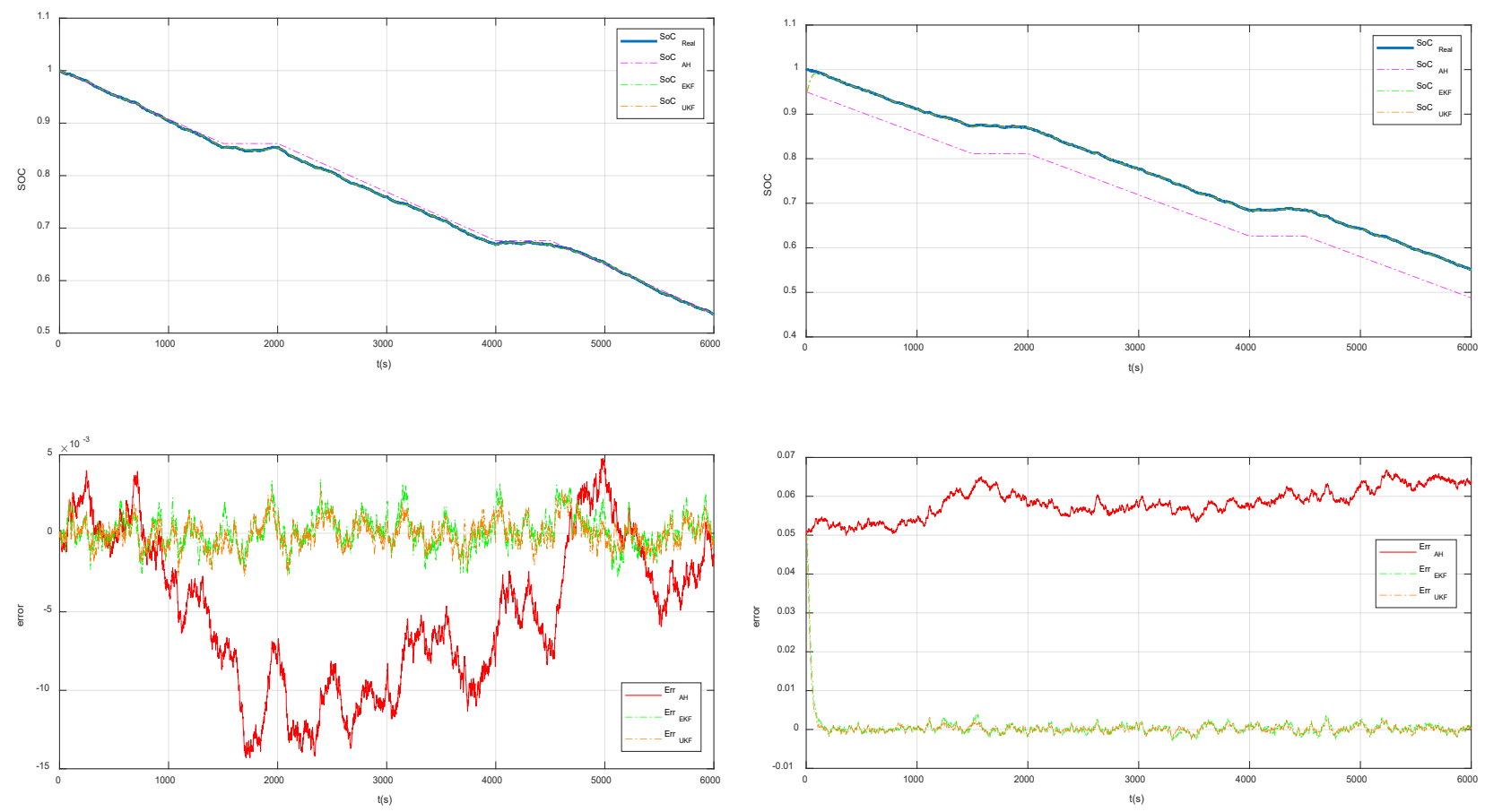

(a) the initial value is accurate with constant current condition(b) deviation initial value with constant current condition 


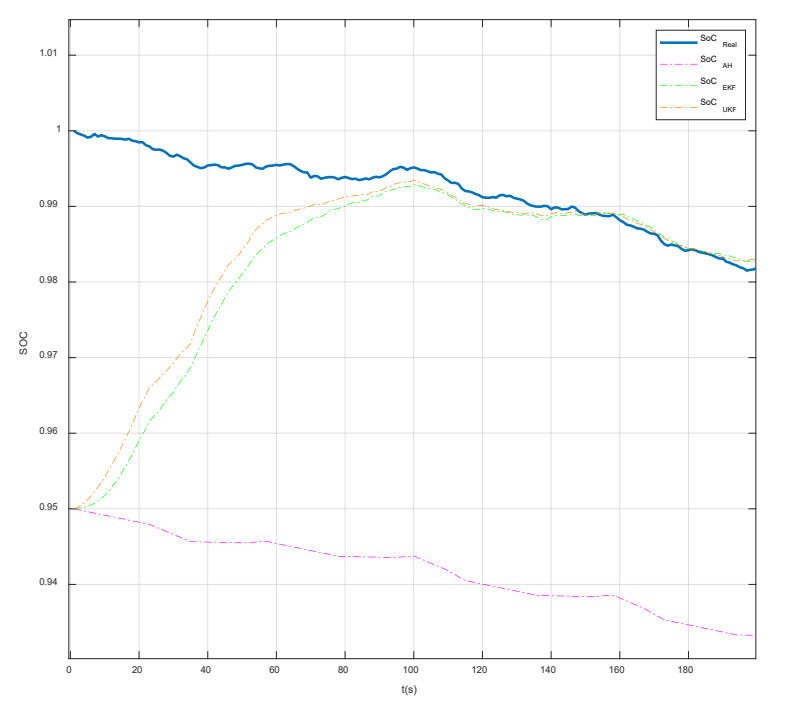

(c) $P_{\text {SOC } 0}=1 \times 10^{-5}$, the initial estimated value is 0.98

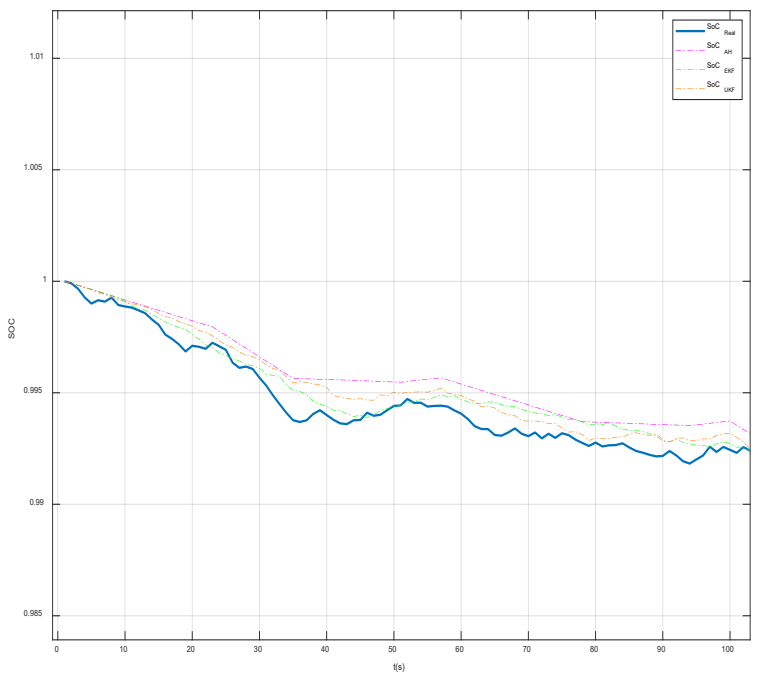

(d) $P_{\text {SOC } 0}=1 \times 10^{-5}$, the initial estimated value is 1

Fig. 9 Different working condition experimental results

As can be seen from the experimental results, as time goes by, the error accumulation effect of Ampere hour integral becomes more and more obvious, and the deviation between the estimated value and the real curve becomes larger and larger. This is mainly because Ampere hour integral method is a one-way estimation method, and there is no feedback correction link, which leads to the phenomenon of error accumulation. However, the estimation curves of differential Kalman filtering and traditional Kalman filtering always follow the real value and fluctuate slightly around it. As can be seen from the error curve, the estimation errors of differential Kalman filtering and traditional Kalman filtering are always within $1.00 \%$, showing good stability. Set the initial estimate value to deviate from the true value, and simulate the case of inaccurate initial value. Here, set the initial estimate value to be 0.99 and the true value to be 1 . Run the simulation model to verify that the output waveform of the algorithm automatically converges to the actual value of the simulated waveform. It can be concluded that differential Kalman filtering and traditional Kalman filtering algorithms can adjust the initial value error automatically. In a short time, the state of charge estimation converges around the real value and still maintains the same estimation accuracy, and the output waveform converges to the actual value curve of the module within a limited sampling period compared with the references [31, 66, 67], which verifies that the improved unscented 
Kalman filtering algotihm and traditional Kalman filtering algorithm have adaptive adjustment ability in the state of initial value error, and state of charge estimation accuracy does not decline.

According to the simulation debugging, the main factor affecting the convergence speed of Kalman filter algorithm is the initial value of error covariance matrix $P$. The convergence speed of the algorithm accelerates with the increase of $P$, but at the same time, it will increase the jitter degree of the algorithm in the initial tracking stage. When the state of $S, P, S_{0}$ become the large initial error covariance matrices, is not allowed for state of charge estimation initial value $\left(S_{0}=0.98\right)$, the condition of differential Kalman filtering and traditional Kalman filtering algorithm can in a very short period of time ( $10 \sim 20$ sampling period $)$ convergence to the true value, but even in the case of initial value accurate, the Kalman filtering will be the initial stage of a tremor and the estimation deviates from the value of the real phenomenon. However, the initial error covariance matrix is large in the case of the same initial estimation deviation value, and the algorithm needs to track to the actual state for more time (80 100 sampling periods). In the case that the initial estimation value is accurate, the algorithm will not deviate at the beginning stage. Therefore, the selection of initial error covariance matrix directly affects the convergence rate of the algorithm and the tracking effect at the beginning.

According to the comparison between the simulation results of differential Kalman filtering and traditional Kalman filtering, it is known that the errors of the two algorithms during the stable tracking period are not much different, and the accuracy is roughly the same. In the case of the same accuracy, the complexity of differential Kalman filtering's algorithm is lower. Comprehensive analysis shows that differential Kalman filtering algorithm based on equivalent circuit model has good stability, accuracy and quick response ability compared with the references [68]. Due to the low complexity of the algorithm, it is suitable for practical lithium battery management system development. 


\section{Conclusions}

A dynamic equivalent model is constructed to represent the working state and output characteristics of lithium batteries in a simple and effective manner and its model parameter identification is realized through hybrid pulse power characteristic experiments. Based on the battery model, an improved differential Kalman filter algorithm is proposed to estimate the battery state of charge. Moreover, the Beijing bus dynamic stress test condition verification experiment shows that the maximum error of state of charge estimation is only 0.022 , which realizes the accurate estimation of ternary lithium battery state of charge, indicating the feasibility and accuracy of this estimation method. It plays an important role in the process analysis and safe use of lithium battery, which has an important advantage in solving small sample, nonlinear and high-dimensional pattern recognition. As mounts of factors affects the state of charge value of lithium battery, so these variables with nonlinear changes are taken as the input parameters. An accurate ternary lithium battery equivalent model is established based on hybrid pulse power characteristic experiment. Combined with differential Kalman filtering algorithm, a filtering program is written in S function to estimate state of charge value. Furthermore, a Beijing bus dynamic stress test condition verification experiment was designed to demonstrate the feasibility and accuracy of the method and its modeling effect is verified for the high-power lithium batteries.

\section{Acknowledgments}

The work was supported by National Natural Science Foundation of China (No. 61801407), China Scholarship Council (No. 201908515099), Sichuan Province Science and Technology Support Program (No. 19ZDYF1098, 2019JDTD0019, 2019YFG0427, 2018GZ0390), Scientific Research Fund of Sichuan (No. 17ZB0453), Teaching Research Project (181zx665, 18gizx11, 18xnsu12). Thanks to the sponsors. CF would like to express his gratitude to PALS for its support.

\section{References}

1. Zhu, J.G., et al., An improved electro-thermal battery model complemented by current dependent parameters for vehicular low temperature application. Applied Energy, 2019. 248: p. 149-161.

2. Zhao, Y., et al., A review on modeling of electro-chemo-mechanics in lithium-ion batteries. Journal of Power Sources, 2019. 413: p. 259-283. 
3. Nandanwar, M. and S. Kumar, A modelling and simulation study of soluble lead redox flow battery: Effect of presence of free convection on the battery characteristics. Journal of Power Sources, 2019. 412: p. 536 -544.

4. Michaelis, C., et al., Electrochemical performance and modeling of lithium-sulfur batteries with varying carbon to sulfur ratios. International Journal of Energy Research, 2019. 43(2): p. 874-883.

5. Zhao, X.C., et al., Electrochemical-thermal modeling of lithium plating/stripping of Li (NiO.6MnO.2Co0.2)O2/Carbon lithium-ion batteries at subzero ambient temperatures. Journal of Power Sources, 2019. 418: p. $61-73$.

6. Zhang, N., et al., A Novel Method for Estimating State-of-Charge in Power Batteries for Electric Vehicles. International Journal of Precision Engineering and Manufacturing, 2019. 20(5): p. 845-852.

7. Meng, X.B., H.X. Li, and H.D. Yang, Evolutionary Design of Spatio-Temporal Learning Model for Thermal Distribution in Lithium-Ion Batteries. Ieee Transactions on Industrial Informatics, 2019. 15(5): p. 2838-2848.

8. Meng, J.H., et al., A Simplified Mode Based State-of-Charge Estimation Approach for Lithium-lon Battery With Dynamic Linear Model. Ieee Transactions on Industrial Electronics, 2019. 66(10): p. 7717-7727.

9. Maiza, M., et al., Evaluating the impact of transport inertia on the electrochemical response of lithium ion battery single particle models. Journal of Power Sources, 2019. 423: p. 263-270.

10. Zhang, B.W., et al., A two-dimensional mathematical model for vanadium redox flow battery stacks incorporating nonuniform electrolyte distribution in the flow frame. Applied Thermal Engineering, 2019. 151: p. $495-505$.

11. Ma, Z.Y., R.X. Yang, and Z.P. Wang, A novel data-model fusion state-of-health estimation approach for lithium-ion batteries. Applied Energy, 2019. 237: p. 836-847.

12. Lyu, Z.Q. and R.J. Gao, A model-based and data-driven joint method for state-of-health estimation of lithium-ion battery in electric vehicles. International Journal of Energy Research, 2019.

13. Lyu, C., et al., In situ monitoring of lithium-ion battery degradation using an electrochemical model. Applied Energy, 2019. 250: p. 685-696.

14. Lu, X., H. Li, and N. Chen, An indicator for the electrode aging of lithium-ion batteries using a fractional variable order model. Electrochimica Acta, 2019. 299: p. 378-387.

15. Dey, S., et al., A Novel Model-Based Estimation Scheme for Battery-Double-Layer Capacitor Hybrid Energy Storage Systems. leee Transactions on Control Systems Technology, 2019. 27(2): p. 689-702.

16. Zelger, C., et al., State of charge indicators for alkaline zinc-air redox flow batteries. Journal of Power Sources, 2019. 424: p. 76-81.

17. Yin, Y.L., et al., New fast charging method of lithium-ion batteries based on a reduced order electrochemical model considering side reaction. Journal of Power Sources, 2019. 423: p. 367-379.

18. Yao, S.G., et al., A dynamic model for discharge research of zinc-nickel single flow battery. Electrochimica Acta, 2019. 307: p. 573-581.

19. Yang, S., et al., Improved constitutive model of the jellyroll for cylindrical lithium ion batteries considering microscopic damage. Energy, 2019. 185: p. 202-212.

20. Yang, N.X., et al., An improved semi-empirical model for thermal analysis of lithium-ion batteries. Electrochimica Acta, 2019. 311: p. 8-20.

21. Dai, X.K., Y. Song, and T.C. Yang, Modelling and region stability analysis of wind turbines with battery energy storage system based on switched system with multi-equilibriums. Transactions of the Institute of Measurement and Control, 2019. 41(6): p. 1519-1527.

22. Dai, H.D., et al., A Novel Estimation Method for the State of Health of Lithium-lon Battery Using Prior Knowledge-Based Neural Network and Markov Chain. leee Transactions on Industrial Electronics, 2019. 66(10): p. 7706-7716.

23. Cui, X.Y., et al., State-of-charge estimation of power lithium-ion batteries based on an embedded micro 
control unit using a square root cubature Kalman filter at various ambient temperatures. International Journal of Energy Research, 2019. 43(8): p. 3561-3577.

24. Chen, Z.H., et al., Particle filter-based state-of-charge estimation and remaining-dischargeable-time prediction method for lithium-ion batteries. Journal of Power Sources, 2019. 414: p. 158-166.

25. Chen, C., et al., State-of-charge estimation of lithium-ion battery using an improved neural network model and extended Kalman filter. Journal of Cleaner Production, 2019. 234: p. 1153-1164.

26. Yang, J.B., et al., Modeling and optimal energy management strategy for a catenary-battery-ultracapacitor based hybrid tramway. Energy, 2019. 183: p. 1123-1135.

27. Yang, F.F., et al., A coulombic efficiency-based model for prognostics and health estimation of lithium-ion batteries. Energy, 2019. 171: p. 1173-1182.

28. Yang, F.F., et al., State-of-charge estimation of lithium-ion batteries based on gated recurrent neural network. Energy, 2019. 175: p. 66-75.

29. Yan, W.Z., et al., A Battery Management System With a Lebesgue-Sampling-Based Extended Kalman Filter. leee Transactions on Industrial Electronics, 2019. 66(4): p. 3227-3236.

30. Yamanaka, T., et al., Modeling lithium ion battery nail penetration tests and quantitative evaluation of the degree of combustion risk. Journal of Power Sources, 2019. 416: p. 132-140.

31. Xie, S.B., et al., Model predictive energy management for plug-in hybrid electric vehicles considering optimal battery depth of discharge. Energy, 2019. 173: p. 667-678.

32. Weber, N., et al., Modeling discontinuous potential distributions using the finite volume method, and application to liquid metal batteries. Electrochimica Acta, 2019. 318: p. 857-864.

33. Wang, X.H., et al., Dependency analysis and degradation process-dependent modeling of lithium-ion battery packs. Journal of Power Sources, 2019. 414: p. 318-326.

34. Wang, S.L., et al., An improved packing equivalent circuit modeling method with the cell-to-cell consistency state evaluation of the internal connected lithium-ion batteries. Energy Science \& Engineering, 2019. 7(2): p. 546-556.

35. Wang, L.M., et al., Study on electrochemical and thermal characteristics of lithium-ion battery using the electrochemical-thermal coupled model. International Journal of Energy Research, 2019. 43(6): p. 2086-2107.

36. Wang, L.M., et al., State of Charge estimation for LiFePO4 battery via dual extended Kalman filter and charging voltage curve. Electrochimica Acta, 2019. 296: p. 1009-1017.

37. Wang, L.B., S. Yin, and J. Xu, A detailed computational model for cylindrical lithium-ion batteries under mechanical loading: From cell deformation to short-circuit onset. Journal of Power Sources, 2019. 413: p. 284-292.

38. von Luders, C., et al., Modeling of lithium plating and lithium stripping in lithium-ion batteries. Journal of Power Sources, 2019. 414: p. 41-47.

39. Velez, P., et al., Modeling of substitutionally modified graphene structures to prevent the shuttle mechanism in lithium-sulfur batteries. Electrochimica Acta, 2019. 309: p. 402-414.

40. Trovo, A., et al., Thermal modeling of industrial-scale vanadium redox flow batteries in high-current operations. Journal of Power Sources, 2019. 424: p. 204-214.

41. Trovo, A., et al., Standby thermal model of a vanadium redox flow battery stack with crossover and shuntcurrent effects. Applied Energy, 2019. 240: p. 893-906.

42. Tian, J.P., R. Xiong, and Q.Q. Yu, Fractional-Order Model-Based Incremental Capacity Analysis for Degradation State Recognition of Lithium-Ion Batteries. leee Transactions on Industrial Electronics, 2019. 66(2): p. 1576-1584.

43. Tang, X.P., et al., A novel framework for Lithium-ion battery modeling considering uncertainties of temperature and aging. Energy Conversion and Management, 2019. 180: p. 162-170. 
44. Tang, X.P., et al., Load-responsive model switching estimation for state of charge of lithium-ion batteries. Applied Energy, 2019. 238: p. 423-434.

45. Stroe, D.I., et al., Electrochemical Impedance Spectroscopy-Based Electric Circuit Modeling of Lithium-Sulfur Batteries During a Discharging State. leee Transactions on Industry Applications, 2019. 55(1): p. 631-637.

46. Stolze, C., M.D. Hager, and U.S. Schubert, State-of-charge monitoring for redox flow batteries: A symmetric opencircuit cell approach. Journal of Power Sources, 2019. 423: p. 60-67.

47. Song, Z.Y., et al., Current Profile Optimization for Combined State of Charge and State of Health Estimation of Lithium Ion Battery Based on Cramer-Rao Bound Analysis. leee Transactions on Power Electronics, 2019. 34(7): p. 7067-7078.

48. Sierra, G., et al., Battery health management for small-size rotary-wing electric unmanned aerial vehicles: An efficient approach for constrained computing platforms. Reliability Engineering \& System Safety, 2019. 182: p. $166-178$.

49. Shukla, V., et al., Modelling high-performing batteries with Mxenes: The case of S-functionalized twodimensional nitride Mxene electrode. Nano Energy, 2019. 58: p. 877-885.

50. Shen, J.N., et al., Accurate State of Charge Estimation With Model Mismatch for Li-lon Batteries: A Joint Moving Horizon Estimation Approach. leee Transactions on Power Electronics, 2019. 34(5): p. 4329-4342.

51. Shahbazi, A.A. and V. Esfahanian, Reduced-order modeling of lead-acid battery using cluster analysis and orthogonal cluster analysis method. International Journal of Energy Research, 2019.

52. Schmitt, T., et al., Zinc electrode shape-change in secondary air batteries: A 2D modeling approach. Journal of Power Sources, 2019. 432: p. 119-132.

53. Saxena, S., et al., Accelerated degradation model for C-rate loading of lithium-ion batteries. International Journal of Electrical Power \& Energy Systems, 2019. 107: p. 438-445.

54. Rosewater, D., et al., Battery Energy Storage State-of-Charge Forecasting: Models, Optimization, and Accuracy. leee Transactions on Smart Grid, 2019. 10(3): p. 2453-2462.

55. Richardson, R.R., M.A. Osborne, and D.A. Howey, Battery health prediction under generalized conditions using a Gaussian process transition model. Journal of Energy Storage, 2019. 23: p. 320-328.

56. Richardson, R.R., et al., Gaussian Process Regression for In Situ Capacity Estimation of Lithium-lon Batteries. leee Transactions on Industrial Informatics, 2019. 15(1): p. 127-138.

57. Ren, H.B., et al., Design and implementation of a battery management system with active charge balance based on the SOC and SOH online estimation. Energy, 2019. 166: p. 908-917.

58. Reinhardt, R., et al., Towards sustainable business models for electric vehicle battery second use: $A$ critical review. Journal of Environmental Management, 2019. 245: p. 432-446.

59. Redondo-Iglesias, E., P. Venet, and S. Pelissier, Efficiency Degradation Model of Lithium-Ion Batteries for Electric Vehicles. Ieee Transactions on Industry Applications, 2019. 55(2): p. 1932-1940.

60. Qin, Y.C., H.C. Hua, and J.W. Cao, Stochastic Optimal Control Scheme for Battery Lifetime Extension in Islanded Microgrid via a Novel Modeling Approach. leee Transactions on Smart Grid, 2019. 10(4): p. 44674475.

61. Poli, F., L.K. Ghadikolaei, and F. Soavi, Semi-empirical modeling of the power balance of flow lithium/oxygen batteries. Applied Energy, 2019. 248: p. 383-389.

62. Perin, I., G.R. Walker, and G. Ledwich, Load Sharing and Wayside Battery Storage for Improving AC Railway Network Performance, With Generic Model for Capacity Estimation, Part 1. leee Transactions on Industrial Electronics, 2019. 66(3): p. 1791-1798.

63. Pang, $\mathrm{H}$., et al., Parameter identification and systematic validation of an enhanced single-particle model with aging degradation physics for Li-ion batteries. Electrochimica Acta, 2019. 307: p. 474-487.

64. Pandzic, H. and V. Bobanac, An Accurate Charging Model of Battery Energy Storage. leee Transactions on 
Power Systems, 2019. 34(2): p. 1416-1426.

65. Panao, M.J.N.O., N.M. Mateus, and G.C. da Graca, Measured and modeled performance of internal mass as a thermal energy battery for energy flexible residential buildings. Applied Energy, 2019. 239: p. 252-267.

66. Xia, Q., et al., A modified reliability model for lithium-ion battery packs based on the stochastic capacity degradation and dynamic response impedance. Journal of Power Sources, 2019. 423: p. 40-51.

67. Wang, Y.J., Z.D. Sun, and Z.H. Chen, Development of energy management system based on a rule-based power distribution strategy for hybrid power sources. Energy, 2019. 175: p. 1055-1066.

68. Xiong, C., et al., Mathematical modeling of the charging process of Li-S batteries by incorporating the sizedependent Li2S dissolution. Electrochimica Acta, 2019. 296: p. 954-963. 